Note

\title{
Comparison of plasma active glucagon-like peptide- 1 (GLP-1) levels assayed with or without plasma extraction in non-diabetic men
}

\author{
Kiyomi Masuda, Kazutaka Aoki and Yasuo Terauchi \\ Department of Endocrinology and Metabolism, Yokohama City University Graduate School of Medicine, Yokohama 236-0004, Japan
}

\begin{abstract}
Recently, interference from cross-reacting peptides in plasma has been recognized as being responsible for inter-subject differences in active glucagon-like peptide-1 (GLP-1) values. An ethanol or solid-phase extraction step could reduce such interference. A working group of the Japan Diabetes Society now recommends the inclusion of an extraction step when measuring active GLP-1 values. We measured the active GLP-1 levels of 200 specimens derived from 10 subjects using both methods and compared the results. The active GLP-1 levels measured by extraction method for 169 specimens with values greater than $2.0 \mathrm{pM}$ tended to be lower than those by direct method. However, the correlation between the GLP-1 levels measured by extraction and direct method was $r=0.9225(p<0.0001)$. In one case, the active GLP-1 level obtained using the extraction method was significantly lower than that obtained using the direct method. Therefore, though there is a good correlation between the two methods, extraction is recommended for more accurate active GLP-1 measurements.
\end{abstract}

Key words: Active glucagon-like peptide-1, Extraction

GLUCAGON-LIKE PEPTIDE-1 (GLP-1) and glucose-dependent insulinotropic polypeptide (GIP) are the two major incretin hormones $[1,2]$ and are secreted from the small intestine. In the past few years, dipeptidyl peptidase-4 (DPP-4) inhibitors, which increase active GLP-1 and GIP by inhibiting DPP-4 enzymatic activity and improve hyperglycemia in a glucosedependent fashion by increasing serum insulin and decreasing serum glucagon levels, have become available for the treatment for patients with diabetes [3, 4].

Recently, the measurement of active GLP-1 levels has become more common in clinical research $[5,6]$. When such measurements were compared among subjects, however, cross-reacting peptides in the plasma were suspected to interfere with the actual GLP-1 levels [7]. An ethanol extraction step or a solid-phase extraction system could reduce such non-specific inter-

Submitted Jan. 8, 2012; Accepted Jan. 31, 2012 as EJ12-0009

Released online in J-STAGE as advance publication Feb. 21, 2012

Correspondence to: Yasuo Terauchi, M.D., Ph.D., Department of Endocrinology and Metabolism, Yokohama City University Graduate School of Medicine, Fuku-ura, Kanazawa-ku, Yokohama 236-0004, Japan. terauchi@yokohama-cu.ac.jp ference in plasma [7]. The extraction procedure for the removal of non-specific immunoactive agents, such as plasma proteins, is relatively complex; however, active GLP-1 levels determined using the extraction procedure are considered to be more accurate. Therefore, the Japan Diabetes Society organized a working group for the standardization of the measurement of incretins. The group recently proposed that an extraction procedure should be performed to provide more accurate values and is presently planning the standardization of a method for the accurate measurement of incretin [8]. However, reports on the measurement of active GLP-1 followed by the preprocessing step are very limited. These conditions prompted us to examine the correlation between active GLP-1 measurements obtained using direct and extraction measurements. In the present study, we measured the active GLP-1 levels in 200 specimens derived from 10 subjects using both the direct and extraction methods and compared the active GLP-1 levels measured using these two methods. We concluded that despite its complexity, the extraction procedure should be recommended to enable more accurate active GLP-1 measurements. 


\section{Materials and Methods}

We measured the active GLP-1 value in a total of 200 samples from 10 subjects in the study [9]. Briefly, we performed four examinations in 10 subjects between May 2010 and September 2010. We had obtained approval for this previous study from the Institutional Ethics Review Committee of Yokohama City University, and informed consent was obtained from each of the subjects prior to the start of the study. Ten non-diabetic men (6 healthy men with normal glucose tolerance, 2 men with an impaired fasting glucose level according to the definition of the World Health Organization, and 2 men with impaired glucose tolerance), aged $39.7 \pm 6.3$ years and with a glycated hemoglobin (HbA1c) level of $5.45 \pm 0.15 \%$ and a body mass index (BMI) of $24.4 \pm 2.8 \mathrm{~kg} / \mathrm{m}^{2}$ were enrolled. The HbA1c (\%) value was estimated as a National Glycohemoglobin Standardization Program (NGSP) equivalent value (\%) calculated by using the formula: HbA1c (\%) = HbA1c (Japan Diabetes Society [JDS]) $(\%)+0.4 \%[10,11]$. Miglitol [50 mg] was administered to each subject according to four different intake schedules (Control: no drug, Intake 1: drug administered just before a meal; Intake 2: drug administered at 30 min after the start of a meal; Intake 3: drug administered at the same time as intake 2 but without eating breakfast). The subjects were randomized to one of the four interventions using a crossover design. All the subjects received a standard breakfast and lunch (773 Kcal; protein:fat:carbohydrate $=1: 1.7: 4.5$ ).

Blood samples were collected before the start of breakfast and at 0 (180 min after the start of breakfast), 30, 60, and 120 min after the start of lunch. Blood samples were collected five times during each examination, and the samples were withdrawn from the catheters directly into BD P700 Blood Collection Tubes (catalogue no. 366473; BD, Franklin Lakes, NJ, USA) containing a DPP-4 inhibitor. The samples were reconstituted and analyzed using an active GLP-1 enzymelinked immunosorbent assay (ELISA) kit (catalogue no. EGLP-35K; Millipore, Billerica, MA, USA). This assay has $100 \%$ cross-reactivity with GLP-1 (7-36) amide and $100 \%$ cross-reactivity with GLP-1 (7-37) but does not react with either GLP-1 (9-36) amide or GLP-1 (9-37). The intra- and interassay coefficients of variation (CV) for the active GLP-1 ELISA were 6.10 $\sim 7.91 \%$ and $3.12 \sim 8.77 \%$, respectively. The recovery was $64.77 \%$ of the expected concentrations for
The C18 column (VARIAN Bond Elut $200 \mathrm{mg}$ )

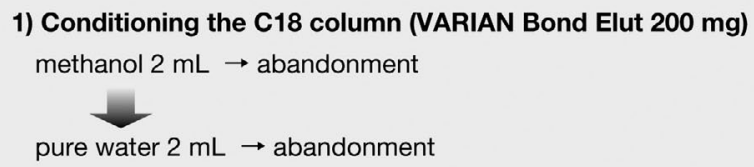

2) Adjustment of specimen for preprocessing: three times dilution GLP-1 specimen $300 \mu \mathrm{L}$

Phosphate buffered saline $600 \mu \mathrm{L}$

3) Column preprocessing process

Conditioning column

Specimen for preprocessing application $900 \mu \mathrm{L}$ Natural fall

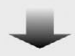

4) Column washing

$10 \%$ methanol $1 \mathrm{~mL} \rightarrow$ abandonment

$10 \%$ methanol $1 \mathrm{~mL} \rightarrow$ abandonment

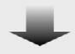

5) GLP-1 elution

$0.5 \%$ ammonia including $75 \%$ methanol. $0.75 \mathrm{~mL} \rightarrow$ elution

$0.5 \%$ ammonia including $75 \%$ methanol. $0.75 \mathrm{~mL} \rightarrow$ elution

Collection of elute in the PP tube $\rightarrow$ total $1.5 \mathrm{~mL}$

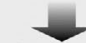

Drying and hardening under nitrogen at $40^{\circ} \mathrm{C}$

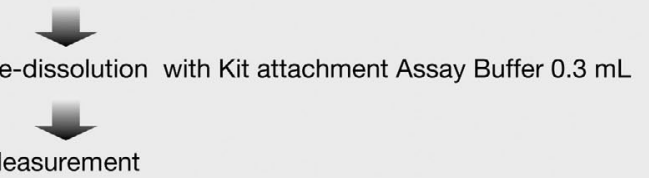

Fig. 1 Extraction procedure before the measurement of plasma levels of active GLP-1.

active GLP-1 added to the plasma. The lowest level of active GLP-1 detectable using this assay was 2.0 pM [12]. We examined the correlation between active GLP-1 levels with values of greater than $2.0 \mathrm{pM}$ with or without plasma extraction. The active GLP-1 levels measured using the extraction method were measured as described in Ref. 13, with slight modifications. The procedure used to extract active GLP- 1 from the plasma is shown in Fig. 1.

The correlation between the active GLP-1 levels measured using the direct and extraction methods was assessed using the Pearson correlation. All statistical analyses were conducted using SPSS for Windows, Japanese version 16.0 (SPSS Institute Inc., Japan). 


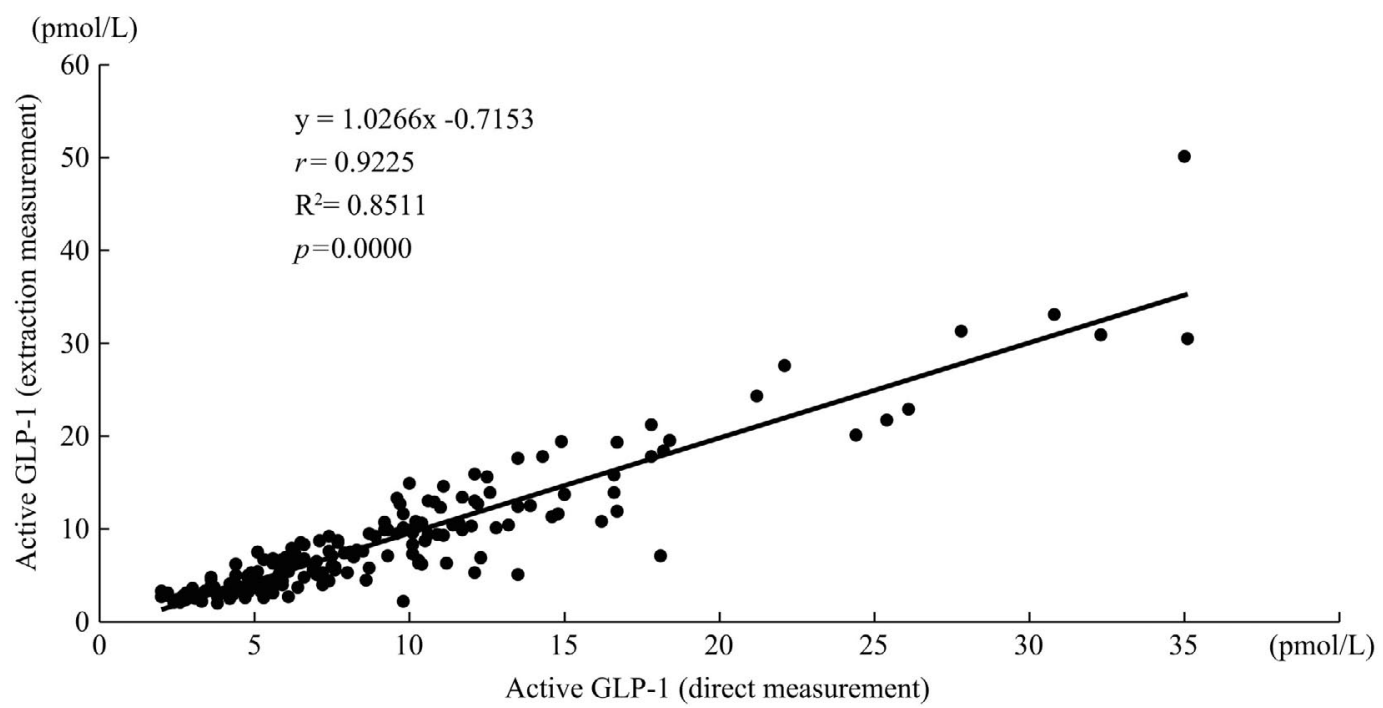

Fig. 2 Correlation between active GLP-1 levels measured directly and those measured after extraction for samples with a GLP-1 level of greater than $2.0 \mathrm{pM}$.

\section{Results}

Thirty-one specimens had active GLP-1 levels that remained below the detection limit (less than $2.0 \mathrm{pM}$ ) throughout the test. Therefore, these 31 specimens were removed from the analysis, and the correlation between the active GLP-1 values measured directly and after extraction for the remaining 169 specimens was determined. Fig. 2 shows the association between the active GLP-1 values measured directly and after extraction for these samples. The correlation was $r=0.9225$ ( $p<$ 0.0001 ). Thus, a strong correlation between the direct and extraction measurements remained, as shown in Fig. 2.

Table 1 shows the plasma active GLP-1 levels measured directly or after extraction for each subject. The active GLP-1 levels measured after extraction tended to be lower than those measured using the direct method $(p=0.20)$; in 1 case (No. 8), the active GLP-1 level measured after extraction was significantly lower than that measured using the direct method.

\section{Discussion}

Recently, extraction has been recognized as an important process for measuring active GLP-1 values more accurately [14]. The reason is thought to be the existence of non-specific interference, which can be removed using ethanol extraction or solid phase extrac-
Table 1 Comparison of plasma active GLP-1 levels measured directly and after extraction for each subject

\begin{tabular}{ccccc}
\hline No. & Direct & Extracted & Difference & $p$ value \\
\hline 1 & $8.70 \pm 1.06$ & $6.81 \pm 1.07$ & 1.89 & 0.10 \\
2 & $8.09 \pm 1.06$ & $6.57 \pm 0.89$ & 1.51 & 0.14 \\
3 & $5.54 \pm 0.68$ & $5.98 \pm 0.85$ & -0.44 & 0.34 \\
4 & $6.24 \pm 0.81$ & $6.98 \pm 1.04$ & -0.74 & 0.28 \\
5 & $8.29 \pm 1.01$ & $6.32 \pm 0.96$ & 1.97 & 0.08 \\
6 & $9.94 \pm 2.14$ & $10.21 \pm 2.24$ & -0.27 & 0.46 \\
7 & $10.03 \pm 1.95$ & $8.97 \pm 1.67$ & 1.06 & 0.34 \\
8 & $6.41 \pm 0.85$ & $4.14 \pm 0.70$ & 2.27 & 0.02 \\
9 & $6.04 \pm 0.73$ & $6.41 \pm 1.00$ & -0.37 & 0.38 \\
10 & $13.24 \pm 1.94$ & $14.69 \pm 2.62$ & -1.45 & 0.33 \\
\hline total & $8.25 \pm 0.44$ & $7.71 \pm 0.48$ & 0.54 & 0.20 \\
\hline
\end{tabular}

Difference: Direct - Extracted

tion [7, 13-15]. Dai et al. reported false overestimations of fasting active GLP-1 levels and improved their results by adding a solid phase extraction step prior to immune-based quantification [15]. LaMarca et al. evaluated the correlation between active GLP-1 levels measured using direct and solid phase extraction methods using different ELISA kits [16]. They reported a good correlation in the active GLP-1 levels measured using the different kits after the removal of substances causing non-specific interference [16]. Yabe et al. recently reported that active GLP-1 values in ethanol- and solid-phase extracted samples showed a good correlation ( $r=0.978, p<0.0001)$, whereas the values of active 
GLP-1 in non-extracted samples showed lower correlations with those values measured in ethanol- or solidphase extracted samples [13]. The active GLP-1 levels measured after extraction tended to be lower than those measured using the direct method, and in 1 case (No. 8), the active GLP-1 level measured after extraction was significantly lower than that measured using the direct method. The measurement of the active GLP-1 levels in those subjects may have been influenced by nonspecific substances. Therefore, the extraction step is recommended to enable the more accurate measurement of active GLP-1 levels, as reported in Ref. 13.

In conclusion, a strong correlation was obtained between the active GLP-1 measurements obtained using the direct measurement and extraction methods. However, an extraction step is recommended to enable more accurate measurements of the active GLP-1 level.

\section{Acknowledgements}

This work was supported in part by Grants-inAid for Scientific Research (B) 19390251 and (B) 21390282 from the Ministry of Education, Culture, Sports, Science and Technology (MEXT) of Japan and a Medical Award from the Japan Medical Association. No potential conflicts of interest relevant to this article exist.

\section{References}

1. Baggio LL, Drucker DJ (2007) Biology of incretins: GLP-1 and GIP. Gastroenterology 132: 2131-2157.

2. Holst JJ (2007) The physiology of glucagon-like peptide 1. Physiological Reviews 87: 1409-1439.

3. Herman GA, Bergman A, Stevens C, Kotey P, Yi B, et al. (2006) Effect of single oral doses of sitagliptin, a dipeptidyl peptidase-4 inhibitor, on incretin and plasma glucose levels after an oral glucose tolerance test in patients with type 2 diabetes. J Clin Endocrinol Metab 91: 4612-4619.

4. Seino Y, Fukushima M, Yabe D (2010) GIP and GLP-1, the two incretin hormones: similarities and differences. J Diabetes Invest 1: 9-23.

5. Vilsbøll T, Krarup T, Sonne J, Madsbad S, Vølund A, et al. (2003) Incretin secretion in relation to meal size and body weight in healthy subjects and people with type 1 and type 2 diabetes mellitus. J Clin Endocrinol Metab 88: 2706-2713.

6. Meier JJ, Nauck MA, Kranz D, Holst JJ, Deacon CF, et al. (2004) Secretion, degradation, and elimination of glucagon-like peptide 1 and gastric inhibitory polypeptide in patients with chronic renal insufficiency and healthy control subjects. Diabetes 53: 654-662.

7. Deacon CF, Holst JJ (2009) Immunoassays for the incretin hormones GIP and GLP-1. Best Pract Res Clin Endocrinol Metab 23: 425-432.

8. http://www.jds.or.jp/jds_or_jp0/uploads/photos/786.pdf

9. Masuda K, Aoki K, Terauchi Y (2011) Effects of miglitol taken just before or after breakfast on plasma glucose, serum insulin, glucagon and incretin levels after lunch in men with normal glucose tolerance (NGT), impaired fasting glucose (IFG) or impaired glucose tol- erance (IGT). J Diabates Invest 2: 435-440.

10. Seino Y, Nanjo K, Tajima N, Kadowaki T, Kashiwagi A, et al. (2010) Report of the Committee on the Classification and Diagnostic Criteria of Diabetes Mellitus. J Diabetes Invest 1: 212-228.

11. Seino Y, Nanjo K, Tajima N, Kadowaki T, Kashiwagi A, et al. (2010) Report of the Committee on the Classification and Diagnostic Criteria of Diabetes Mellitus. Diabetol Int 1: 2-20.

12. Glucagon-Like Peptide-1 (Active) ELISA Kit 96-Well Plate (Cat. \# EGLP-35K). Copyright 2008 Millipore Corporation, Billerica, MA, U.S.A

13. Yabe D, Watanabe K, Sugawara K, Kuwata H, Kitamoto $\mathrm{Y}$, et al. (2012) Comparison of incretin immunoassays with or without plasma extraction: Incretin secretion in Japanese patients with type 2 diabetes. $J$ Diabates Invest 3: 70-79.

14. Yabe D, Kuroe A, Lee S,Watanabe K, Hyo T, et al. (2010) Little enhancement of meal-induced glucagonslike peptide 1 secretion in Japanese: Comparison of type 2 diabetes patients and healthy controls. J Diabates Invest 1: 56-59.

15. Dai H, Gustavson SM, Preston GM, Eskra JD, Calle $\mathrm{R}$, et al. (2008) Non-linear increase in GLP-1 levels in response to DPP-IV inhibition in healthy adult subjects. Diabetes Obes Metab 10: 506-513.

16. LaMarca CJ, Perregaux DG, Preston GM, Shaw CM, Gao P (2009) Comparison and validation of multiple commercially available methods for active GLP-1 quantification : sample extraction is a ubiquitous requirement. In : ENDO 2009. Washington,D.C. 